\title{
ISSUES ON RADIATIVELY INDUCED LORENTZ AND CPT VIOLATION IN QUANTUM ELECTRODYNAMICS
}

\author{
W.F. CHEN \\ Department of Mathematics and Statistics, University of Guelph \\ Guelph, Ontario, Canada N1G 2W1 \\ E-mail: wchen@uoguelph.ca
}

\begin{abstract}
Various ambiguous results on radiatively induced Lorentz and CPT violation in quantum electrodynamics with a modified fermionic sector are reviewed and possible explanations for this ambiguity appearing in the literature are commentated. Furthermore, joint between stringent limit from astrophysical observation and theoretical prediction on Lorenz and CPT violation is discussed.
\end{abstract}

\section{Introduction}

Lorentz symmetry is algebraic foundation of the theory of special relativity. Nearly one hundred years the theory of special relativity keeps the status as a cornerstone of modern physics and has been supported by numerous high energy physics experiments and astrophysical observation. However, physics is a science born out of experimental observation. With the availability of higher precision experimental or observational data, it is conceivable that even the most fundamental principles may someday have to be modified or even abandoned. There are quite a number of such examples in the history of physics. It is partly in this spirit that an investigation on the possible breaking of Lorentz symmetry is not fantastic.

In fact, the spontaneous breaking of Lorentz symmetry is a natural consequence of string theory. If the Standard Model is considered as the low-energy limit of a more fundamental theory constructed from string, the spontaneous breaking of Lorentz symmetry can occur naturally since string theory generally involves interactions that make a Lorentz tensor get non-zero vacuum expectation valuel.

A straightforward reason of considering Lorentz and CPT violation in quantum electrodynamics (QED) was from astrophysical observation. A lopsided analysis on the polarized electromagnetic radiation emitted by distant radio galaxies revealed that the universe may present cosmological anisotropy in electromagnetic wave propagation 2 . Moreover, the analysis suggested that this chiral effect can be well described at lower derivative expansion by a modified classical electrodynamics proposed a decade ago $\mathbf{B}$,

$$
S=\int d^{4} x\left(-\frac{1}{4} F_{\mu \nu} F^{\mu \nu}+\frac{1}{2} \epsilon^{\mu \nu \lambda \rho} k_{\mu} F_{\nu \lambda} A_{\rho}\right) .
$$

The first term of (11) is the familiar Maxwell term, and the second one is called the ChernSimons-like (CS) term,

$$
\mathcal{L}_{\mathrm{CS}}=\frac{1}{2} \epsilon^{\mu \nu \lambda \rho} k_{\mu} F_{\nu \lambda} A_{\rho}
$$

which explicitly violate Lorentz and discrete CPT symmetries since $k_{\mu}$ is certain background constant vector in four-dimensional space-time. Despite that a more rigorous analysis on the astrophysical observation data has excluded the polarization effect of electromagnetic

pascos01: submitted to Rinton on October 30, 2018 
wave in propagating from the distant radio sourcest, this still provides a promising way to observe a violation of Lorentz and CPT symmetries in nature, and hence stimulates an effort to explore a possible Lorentz and CPT violating mechanism theoretically.

Furthermore, a $S U(3) \times S U(2) \times U(1)$ standard model with explicit Lorentz and CPT violating extension had been constructed and hence a quantitative physical theory of studying Lorentz and CPT violation was furnished 5 . Predictions on the possible Lorentz and CPT violation from this extended Standard Model can be tested by high-precision measurements in numerous existing experiments and possibly in next generation accelerator 6 .

In this talk I shall concentrate on a typical quantum field theory problem, namely, whether the CPT-odd pure photon term in electrodynamics, i.e. the CS term shown in Eq. (2), can be induced from quantum correction with a modified fermionic sector

$$
\mathcal{L}_{\text {fermion }}=\bar{\psi}\left(i \not \partial-e \not A-\not b \gamma_{5}-m\right) \psi,
$$

where $b_{\mu}$ is a constant prescribed four-vector. The new introduced gauge invariant interaction term between constant vector $b_{\mu}$ and axial vector current $j_{\mu}^{5}(x)=\bar{\psi} \gamma_{\mu} \gamma_{5} \psi$ violates Lorenz and CPT symmetries explicitly, since $b_{\mu}$ picks up a fixed direction in space-time. If the CS term can be induced from the radiative correction with the coefficient $k_{\mu} \propto b_{\mu}$, then a constraint on $\mathcal{L}_{C S}$ from astrophysical observation will restrict a possible Lorentz and $\mathrm{CPT}$ violation in the fermionic sector.

In section 2 we shall review various results on radiatively induced CS term, and then analyze the possible origin for this ambiguity in section 3. Finally we summarize and discuss the joint of astrophysical observation with theoretical prediction on Lorentz and $\mathrm{CPT}$ violation and some relevant problems.

\section{Various Controversial and Ambiguous Results on Radiatively Induced Chern-Simons Term}

As a general procedure, the quantum effective action can be obtained by integrating out fermionic fields,

$$
\begin{aligned}
e^{i \Gamma[A, b]} & =\int D \bar{\psi} D \psi e^{i \int d^{4} x \bar{\psi}\left(i \not \partial-e A-\not \gamma_{5}-m\right) \psi} \\
& =\operatorname{det}\left(i \not \partial-e A-\not \partial \gamma_{5}-m\right) ; \\
\Gamma[A, b] & =-i \operatorname{Tr} \ln \left(i \not \partial-e \not A-\not b \gamma_{5}-m\right) .
\end{aligned}
$$

The radiatively induced Chern-Simons term will be $b$-linear and parity-odd part of above effective action.

It is well known that $\Gamma[A, b]$ or equivalently the relevant fermionic determinant cannot be evaluated exactly. A perturbative expansion or certain approximation must be utilized and a use of regularization scheme must be made in the calculation. At first sight, the evaluation of CS term in the quantum effective action is a typical and simple quantum field theory problem. However, the concrete calculation turned out to be rather non-trivial and the result presented remarkable ambiguities: distinct relations between $k_{\mu}$ and $b_{\mu}$ can yield depending on concrete calculation schemes. The various ambiguous results are listed in the following:

$$
k_{\mu}=0
$$

pascos01: submitted to Rinton on October 30, 2018 
Coleman and Glashow argued first that the CS term cannot be generated 7 . They considered that the axial vector current $j_{\mu}^{5}(x)=\bar{\psi}(x) \gamma_{\mu} \gamma_{5} \psi(x)$ should keep gauge invariant in the quantum theory at any momentum or equivalently at any space-time point. Since $\left\langle j_{\mu}^{5}(x)\right\rangle=\delta \mathcal{L}(x) / \delta b_{\mu}$, this hypothesis is actually equivalent to the requirement that the Lagrangian density corresponding to the quantum effective action should be gauge invariant. Thus, based on this requirement, the CS term cannot be generated since its Lagrangian density is explicitly not invariant under gauge transformation $A_{\mu} \rightarrow A_{\mu}+\partial_{\mu} \Lambda$. Furthermore, Bonneau studied the renormalization of an extended QED including the CS term (2) and the modified fermionic sector (3). He found that Ward identities and the renormalization conditions determine uniquely the absence of CS term from quantum corrections. In fact, when deriving Ward identities, Bonneau introduced external source fields for the axial vector current and the CS term, so the Ward identities he derived actually impose gauge invariance on Lagrangian density and hence coincide with the "no-go theorem" requirement argued by Coleman and Glashowl. Recently, Adam and Klinkhamer put forward an independent line of reasoning the vanishing of radiatively induced CS term rather than gauge symmetry 3 . They argued that if the perturbative expansion near $b^{2}=0$ is valid and further the gauge field $A_{\mu}$ is regarded as a quantized dynamical field rather than an external background field as Bonneau did, the presence of CS term with a purely time-like coefficient may violate the causality principle of a quantum field theory. This argument thus excluded the radiative induction of CS term. In addition, explicit perturbative calculations in Pauli-Villars regularization vanish.

$$
k_{\mu}=\frac{3 e^{2}}{16 \pi^{2}} b_{\mu}
$$

However, Jackiw and Kostelecký thought that since $j_{\mu}^{5}(x)$ only couples with a constant 4 -vector $b_{\mu}$, it is true to require only that $j_{\mu}(x)$ with zero-momentum (i.e. $\int d^{4} x j_{\mu}^{5}(x)$ ) is gauge invariant at quantum level. Since $\left\langle\int d^{4} x j_{\mu}^{5}(x)\right\rangle=\delta S / \delta b_{\mu}$, this statement is equivalent to the requirement that the quantum effective action should be gauge invariant. Thus the dynamical generation of CS term can escape from above "no-go theorem" conjectured by Coleman and Glashow, since the action of CS term is gauge invariant. Based on this observation, Jackiw and Kostelecký calculated the $b$-linear part of the one-loop vacuum polarization tensor with $b_{\mu}$-exact propagator. They ingeniously manipulated the linear divergent term in the loop integration by writing it into a finite term plus an external momentum derivative term, and then arrived at the finite result (6). Actually, this result was obtained earlier by Chung and $\mathrm{Oh}$ in calculating the one-loop effective action Eq. (4) in terms of dimensional_regularizatoion plus derivative expansion 11 . Furthermore, Pérez-Victoria 12 and Chung 13 proved through an explicit calculation on the parity-odd part of one-loop vacuum polarization tensor at zero external momentum that the result (6) stands to any order of $b_{\mu}$.

$$
k_{\mu}=C b_{\mu}, \quad C \text { being an arbitrary constant. }
$$

pascos01: submitted to Rinton on October 30, 2018 
It was first realized by Jackiw that the perturbative ambiguity for radiatively induced CS term can be revealed quantitatively in a new developed regularization method called differential regularization 14 . This new calculation technique works for a Euclidean field theory in coordinate space. Its invention is based on the observation that the shortdistance singularity representing the UV divergence of a primitively divergent Feynman diagram prevent the amplitude from having a Fourier transform into momentum space. So one can regulate such an amplitude by writing its singular term as a derivative of another function, which has a well defined Fourier transform, then performing Fourier transform into momentum space through partial integration and discarding the surface term. In this way one can directly arrive at a renormalized amplitude. The great advantages of this regularization over conventional regularization methods lie in that it does not modify the original classical action and in particular, it does not impose or violate gauge symmetry in the process of calculation. Only at the end of calculation, one can get the preferred symmetry by an appropriate choice on indefinite renormalization scales. This is the reason why differential regularization can clearly show the perturbative ambiguity. The $b$-linear part of one-loop vacuum polarization tensor in differential regularization reads 15

$$
\begin{aligned}
\Pi_{\mu \nu}^{(b)}(x)= & \frac{m^{2}}{2 \pi^{4}} b_{\lambda} \epsilon_{\lambda \mu \nu \rho}\left\{-2\left[\frac{\partial}{\partial x_{\rho}}\left(\frac{K_{1}(m x)}{x}\right)^{2}-\frac{\partial}{\partial x_{\rho}}\left(\frac{K_{1}(m x)}{x}\right)^{2}\right]\right. \\
& \left.+m \frac{\partial}{\partial x_{\rho}}\left[\frac{K_{1}(m x) K_{0}(m x)}{x}\right]\right\} \\
= & \frac{m^{2}}{2 \pi^{4}} b_{\lambda} \epsilon_{\lambda \mu \nu \rho}\left\{-\frac{1}{2 m^{2}} \frac{\partial}{\partial x_{a}}\left[\partial^{2}\left(\frac{\ln x^{2} M_{1}^{2}}{x^{2}}\right)-\partial^{2}\left(\frac{\ln x^{2} M_{2}^{2}}{x^{2}}\right)\right]\right. \\
& \left.+m \frac{\partial}{\partial x_{\rho}}\left[\frac{K_{1}(m x) K_{0}(m x)}{x}\right]\right\} \\
= & \frac{1}{2 \pi^{4}} b_{\lambda} \epsilon_{\lambda \mu \nu a}\left\{4 \pi^{2} \ln \frac{M_{1}}{M_{2}} \frac{\partial}{\partial x_{\rho}} \delta^{(4)}(x)+m^{3} \frac{\partial}{\partial x_{\rho}}\left[\frac{K_{1}(m x) K_{0}(m x)}{x}\right]\right\}
\end{aligned}
$$

where we have used the asymptotic expansion of the first-order modified Bessel function of the second kind near $x^{2} \sim 0$,

$$
\frac{K_{1}(m x)}{x} \stackrel{x^{2} \sim 0}{\longrightarrow} \frac{1}{m x^{2}}+\frac{1}{4} m \ln \left(m^{2} x^{2}\right)+\cdots,
$$

and the fact that the UV divergence is only contained in the leading term as well as a typical differential regularization identity,

$$
\left(\frac{1}{x^{4}}\right)_{R}=-\frac{1}{4} \partial^{2}\left[\frac{\ln \left(x^{2} M^{2}\right)}{x^{2}}\right] .
$$

The corresponding Fourier transform is thus

$$
\begin{aligned}
\Pi_{\mu \nu}^{(b)}(p)= & \int d^{4} x e^{-i p \cdot x} \Pi_{\mu \nu}(x)=\frac{2}{\pi^{2}} b_{\lambda} \epsilon_{\lambda \mu \nu \rho} i p_{\rho}\left[\ln \frac{M_{1}}{M_{2}}\right. \\
& \left.+\frac{m}{4 p \sqrt{1+p^{2} /\left(4 m^{2}\right)}} \ln \frac{\sqrt{1+p^{2} /\left(4 m^{2}\right)}+p /(2 m)}{\sqrt{1+p^{2} /\left(4 m^{2}\right)}-p /(2 m)}\right],
\end{aligned}
$$


and the CS term is relevant to above polarization tensor at low-energy limit,

$$
\left.\Pi_{\mu \nu}^{(b)}(p)\right|_{p^{2}=0}=\frac{2 i}{\pi^{2}} \epsilon_{\rho \mu \nu \lambda} b_{\rho} p_{\lambda}\left(\ln \frac{M_{1}}{M_{2}}+\frac{1}{4}\right) .
$$

Since $M_{1}$ and $M_{2}$ are two arbitrary renormalization scales, the above result shows that the relation between $k_{\mu}$ and $b_{\mu}$ is completely arbitrary.

Furthermore, the same conclusion was drawn by Chung 16 through an analysis on the non-invariance of path integral measure under axial vector gauge transformation as Fujikawa's method of evaluating chiral anomaly, and then an explicit calculation on the $b$-linear part of the vacuum polarization tensor with the action manifesting the non-invariance under axial vector gauge transformation.

$$
k_{\mu}=\frac{e^{2}}{8 \pi^{2}} b_{\mu}
$$

It was found by Chan 17 , when adopting the covariant derivative expansion 18 to evaluate the anomalous contribution to the effective action (14), that due to the noncommutativity of the operators $\partial$ and $A_{\mu}(x)$ there arises a non-Feynman diagram contribution to the $b$-linear part of the vacuum polarization tensor. This additional term looks quite exotic from the viewpoint of perturbation theory and it seems to represent a somehow non-perturbative contribution. As a consequence, the result (6) was modified to $e^{2} /\left(8 \pi^{2}\right) b_{\mu}$. The basic idea of covariant derivative expansion is to express local quantum effective Lagrangian in powers of gauge covariant derivative $\Pi_{\mu}=i \partial_{\mu}-e A_{\mu}$ rather than in powers of $i \partial_{\mu}$ and $A_{\mu}$ separately 18 . The remarkable difference between (6) and (13) and the feature of covariant derivative expansion motivated us to re-calculate the $b$-linear part of the effective action (1) in the technique of Schwinger's constant field approximation 19 since this method shares the same feature as covariant derivative expansion. The essence of this method is converting the calculation of an effective action as (4) into solving a harmonic oscillator problem in non-relativistic quantum mechanics. It is worth to mention that this is an evergreen method and has been utilized in many aspects such as computation of gravitational anomaly in $4 n+2$ dimensions 20 , investigation on the dynamical violation of parity and gauge symmetries in three dimensions21 and determining the low-energy effective action of $N=2$ supersymmetric Yang-Mills theorye2 etc. We found that if the following trace condition is satisfied,

$$
\lim _{x^{\prime} \rightarrow x} \frac{\left(x-x^{\prime}\right)_{\mu}\left(x-x^{\prime}\right)_{\nu}}{\left(x-x^{\prime}\right)^{2}}=\lim _{x^{\prime} \rightarrow x} \frac{1}{\int d^{4} x^{\prime}} \int d^{4} x^{\prime} \frac{\left(x-x^{\prime}\right)_{\mu}\left(x-x^{\prime}\right)_{\nu}}{\left(x-x^{\prime}\right)^{2}}=\frac{1}{4} g_{\mu \nu},
$$

then the same result as in covariant derivative expansion can be reproduced 23 ,

$$
\begin{aligned}
\left\langle J_{\mu}(x)\right\rangle= & \frac{\delta \Gamma_{\mathrm{CS}}}{\delta A_{\mu}(x)}=\frac{e^{2}}{4 \pi^{2}}\left\{\exp \left[-i e \int_{x^{\prime}}^{x} d y^{\rho} A_{\rho}(y)\right] \frac{m K_{1}\left(\left[-m^{2}\left(x-x^{\prime}\right)^{2}\right]^{1 / 2}\right.}{\left[-m^{2}\left(x-x^{\prime}\right)^{2}\right]^{1 / 2}}\right. \\
& \left.\times\left(x-x^{\prime}\right)_{\mu}\left(x-x^{\prime}\right)_{\rho} b_{\nu} \epsilon^{\rho \nu \alpha \beta} F_{\alpha \beta}\right\}\left.\right|_{x^{\prime} \rightarrow x} \\
= & -\frac{e^{2}}{16 \pi^{2}} \epsilon^{\mu \nu \lambda \rho} b_{\nu} F_{\lambda \rho}, \\
\Gamma_{\mathrm{CS}}= & \frac{e^{2}}{16 \pi^{2}} \int d^{4} x \epsilon^{\mu \nu \lambda \rho} b_{\mu} A_{\nu} F_{\lambda \rho} .
\end{aligned}
$$


However, it should be emphasized that the limit given in Eq. (14) has a potential ambiguity and the general result will be that $\lim _{x \rightarrow 0} x_{\mu} x_{\nu} / x^{2}=C g_{\mu \nu}$, thus the induced CS term is actually ambiguous. Furthermore, this ambiguous result was confirmed by Chungs to any order of $b_{\mu}$ in the same method24.

\section{Possible Origin of Ambiguity}

Four finite but entirely different results on radiatively induced CS term are shown in last section. The reason behind this ambiguity should be unearthed. Two convincing explanations has been proposed by Sitenko 25 and Pérez-Victoria27. The former emphasized the calculation technique cause, while the latter indicated a theoretical origin.

Let us first look at the explanation proposed by Sitenkd25, which concerns with two different formulations of the quantum effective action (住) adopted by Chaichian et ale2 and Chung et al11,

$$
\begin{aligned}
& \Gamma^{(1)}=-i \operatorname{Tr} \ln (i \not \partial-\not A-m)+i \int_{0}^{1} d z \operatorname{Tr}\left[\left(i \not \partial-\not A-z \gamma^{5} \not b-m\right)^{-1} \gamma^{5} \not b\right], \\
& \Gamma^{(2)}=-i \operatorname{Tr} \ln \left(i \not \partial-\gamma^{5} \not b-m\right)+i \int_{0}^{1} d z \operatorname{Tr}\left[\left(i \not \partial-z \not A-\gamma^{5} \not b-m\right)^{-1} \not A\right] .
\end{aligned}
$$

Their $b$-linear sectors read

$$
\begin{aligned}
& i \int_{0}^{1} d z \operatorname{Tr}\left[\left(i \not \partial-A-z \gamma^{5} \not b-m\right)^{-1} \gamma^{5} \not\right]_{(b)}=\frac{1}{16 \pi^{2}} b_{\mu} \int d^{4} x \epsilon^{\mu \nu \lambda \rho} F_{\nu \lambda} A_{\rho} \\
+ & \frac{1}{8 \pi^{2}} b_{\mu} I_{\nu} \int d^{4} x \epsilon^{\mu \nu \lambda \rho} F_{\lambda \rho}+\frac{1}{8 \pi^{2}} I_{\alpha \beta}\left(g^{\beta \nu} \epsilon^{\mu \alpha \rho \lambda}+g^{\beta \lambda} \epsilon^{\mu \nu \rho \alpha}\right) b_{\mu} \int d^{4} x A_{\nu} \partial_{\rho} A_{\lambda}, \\
& i \int_{0}^{1} d z \operatorname{Tr}\left[\left(i \not \partial-z A-\gamma^{5} \not b-m\right)^{-1} A\right]_{(b)}=\frac{3}{32 \pi^{2}} b_{\mu} \int d^{4} x \epsilon^{\mu \nu \lambda \rho} F_{\nu \lambda} A_{\rho} \\
+ & \frac{1}{8 \pi^{2}} I_{\alpha \beta}\left(g^{\beta \nu} \epsilon^{\mu \alpha \rho \lambda}+g^{\beta \lambda} \epsilon^{\mu \nu \rho \alpha}+g^{\beta \rho} \epsilon^{\mu \nu \rho \alpha}\right) b_{\mu} \int d^{4} x A_{\nu} \partial_{\rho} A_{\lambda},
\end{aligned}
$$

where

$$
\begin{aligned}
I_{\mu} & \equiv \frac{1}{i \pi^{2}} \int d^{4} k \frac{k_{\mu}}{\left(k^{2}-m^{2}\right)^{2}}, \\
I_{\mu \nu} & \equiv \frac{1}{i \pi^{2}} \int d^{4} k \frac{4 k_{\mu} k_{\nu}-k^{2} g_{\mu \nu}}{\left(k^{2}-m^{2}\right)^{3}}=\frac{1}{2} g_{\mu \nu}-\frac{1}{i \pi^{2}} \int d^{4} k \frac{\partial}{\partial k^{\mu}}\left[\frac{k_{\nu}}{\left(k^{2}-m^{2}\right)^{2}}\right]
\end{aligned}
$$

are two momentum integrals with superficially linear and logarithmic divergence, respectively. Due to the explicit breaking of Lorentz symmetry, one cannot naively put $I_{\mu}=0$ and use the formula $\int d^{n} k k_{\mu} k_{\nu} f\left(k^{2}\right)=1 / n g_{\mu \nu} \int d^{n} k k^{2} f\left(k^{2}\right)$. The approaches of regularization schemes manipulating these two divergent integrals lead to above finite ambiguities on the radiatively induced CS term 25 :

- If a regularization scheme defines $I_{\mu}=0$ and imposes the trace condition $g^{\mu \nu} I_{\mu \nu}=0$, this actually yields $I_{\mu \nu}=0$ due to the fact $I_{\mu \nu} \propto g_{\mu \nu}$, then the $b$-linear parts of $\Gamma^{(1)}$ and $\Gamma^{(2)}$ will yield the results obtained by Jackiw et al and Chan et al, respectively,

$$
\Gamma_{\mathrm{CS}}^{(1)}=\frac{1}{16 \pi^{2}} b_{\mu} \int d^{4} x \epsilon^{\mu \nu \lambda \rho} F_{\nu \lambda} A_{\rho} ; \quad \Gamma_{\mathrm{CS}}^{(2)}=\frac{3}{32 \pi^{2}} b_{\mu} \int d^{4} x \epsilon^{\mu \nu \lambda \rho} F_{\nu \lambda} A_{\rho} .
$$

pascos01: submitted to Rinton on October 30, 2018 
- In a regularization scheme defining $I_{\mu}=0$ and $I_{\mu \nu}=1 / 2 g_{\mu \nu}$ (i.e. omitting the surface term of $\left.I_{\mu \nu}\right)$, the conclusion argued by Coleman and Glashow will be reproduced,

$$
\Gamma_{\mathrm{CS}}^{(1)}=\Gamma_{\mathrm{CS}}^{(2)}=0 .
$$

- If defining the one-loop quantum effective action as an arbitrary combination of (17) and (18) and choosing a regularization scheme imposing $I_{\mu}=I_{\mu \nu}=0$, one can get the result obtained by Chen in differential regularization,

$$
\Gamma_{\mathrm{CS}}=(1-c) \Gamma_{\mathrm{CS}}^{(1)}+c \Gamma_{\mathrm{CS}}^{(2)}=\frac{2+c}{32 \pi^{2}} b_{\mu} \int d^{4} x \epsilon_{\mu \nu \lambda \rho} F_{\nu \lambda} A_{\rho} \equiv C b_{\mu} \int d^{4} x \epsilon_{\mu \nu \lambda \rho} F_{\nu \lambda} A_{\rho} .(24)
$$

The above explanation shows that the origin for the finite ambiguous CS term lies in the inequivalence between two formulations (17) and (18) of the quantum effective action (4) and the ambiguity of a regularization method in manipulating logrithmically and linearly divergent loop momentum integrals.

Pérez-Victoria 27 put forward another explanation through revealing a relation between the radiatively induced CS coefficient and triangle chiral anomaly via an intermediate model having spontaneous breaking of Lorentz and CPT symmetries. The fermionic sector of this model takes following forme27,

$$
\mathcal{L}_{\text {fermion }}^{\prime}=\bar{\psi}\left(i \not D-m+\frac{\widetilde{b}}{\Lambda} \gamma_{5} \not \partial \phi+i c \gamma_{5} \phi+\frac{d}{\Lambda} \phi^{2}\right) \psi,
$$

where $\phi$ is a real pseudoscalar field (i.e. an axion), $\Lambda$ is certain large scale and $\widetilde{b}, c$ and $d$ are indefinite parameters. In the choice that

$$
c=d=0,\langle\phi\rangle=\frac{\Lambda}{\widetilde{b}} b_{\mu} x^{\mu},
$$

the above model will restore the fermionic Lagrangian (3).

As initially argued by Coleman and Glashowd, the radiatively induced CS coefficient $\widetilde{k}$ in the model (25) can be defected by evaluating the quantum vertex $\Gamma_{\mu \nu}(p, q)$ composed of one axion and two photons2 2 , i.e., the 1PI part of the correlation function $\left\langle A_{\mu}(p) A_{\nu}(q) \phi(-p-\right.$ $q)\rangle$,

$$
\Gamma^{\mu \nu}(p, q)=\epsilon^{\mu \nu \lambda \rho} p_{\lambda} q_{\rho} C(p, q), \quad \widetilde{k}=-\frac{\Lambda}{2} C(0,0) .
$$

The Lagrangian (25) shows that the $\widetilde{b}$-linear part of $\Gamma_{\mu \nu}(p, q)$ is explicitly related to the chiral triangle amplitude $V^{\mu \nu \rho}(p, q)=\left\langle j^{\mu}(p) j^{\nu}(q) j_{5}^{\rho}(-p-q)\right\rangle$,

$$
\Gamma_{\widetilde{b}}^{\mu \nu}(p, q)=\frac{\widetilde{b}}{\Lambda} e^{2}\left(p_{\rho}+q_{\rho}\right) V^{\mu \nu \rho}(p, q) .
$$

It is well known that $V^{\mu \nu \rho}$ satisfies the celebrated anomalous Ward identity,

$$
\left(p_{\rho}+q_{\rho}\right) V^{\mu \nu \rho}(p, q)=2 i m V^{\mu \nu}(p, q)+\epsilon^{\mu \nu \lambda \rho} p_{\lambda} q_{\rho} \mathcal{A},
$$

where $\mathcal{A}$ is the chiral anomaly coefficient. Further, the tensor structure of the canonical term $V^{\mu \nu}(p, q)$, which comes from an explicit breaking of chiral symmetry by fermionic mass term, takes the following form,

$$
V^{\mu \nu}(p, q)=\left\langle j^{\mu}(p) j^{\nu}(q) j_{5}(-p-q)\right\rangle=\epsilon^{\mu \nu \lambda \rho} p_{\lambda} q_{\rho} V(p, q), \quad j_{5} \equiv \bar{\psi} \gamma_{5} \psi,
$$


Eqs (27)- (30) establish a relation among the form factor $C_{\widetilde{b}}(p, q)$ of the $\widetilde{b}$-linear sector of $\Gamma^{\mu \nu}(p, q)$, the form factor $V(p, q)$ of the canonical term in the anomalous Ward identity (29) and the anomaly coefficient 27 ,

$$
C_{\widetilde{b}}=\frac{\widetilde{b}}{\Lambda} e^{2}[2 m V(p, q)+\mathcal{A}] .
$$

The canonical term $V^{\mu \nu}(p, q)$ in the anomalous Ward (29) identity is finite and unambiguous. A comparison $\left\langle j^{\mu}(p) j^{\nu}(q) j_{5}(-p-q)\right\rangle$ with $\Gamma_{\mu \nu}(p, q)$ shows that $V^{\mu \nu}(p, q)$ is actually equal to the $c$-linear part of $\Gamma^{\mu \nu}(p, q)$. It turned out that the $c$-linear part of $\Gamma^{\mu \nu}(p, q)$ could be easily calculated since it is convergent27,

$$
\widetilde{k}_{c}=-\frac{c e^{2} \Lambda}{2} V(0,0)=\frac{c e^{2} \Lambda}{8 \pi^{2} m} .
$$

An insertion of Eqs. (27) and (32) into (31) immediately lead to a relation among $k_{\tilde{b}}$, i.e. the coefficient of $\widetilde{b}$-linear part of $\Gamma^{\mu \nu}(p, q)$ defined on the mass-shell of a photon, the parameter $\widetilde{b}$ and anomaly coefficient $\mathcal{A}$,

$$
\widetilde{k}_{\widetilde{b}}=e^{2} \widetilde{b}\left(\frac{1}{4 \pi^{2}}-\frac{1}{2} \mathcal{A}\right) .
$$

Upon choosing the parameters shown in (26), one can find a relatiop between radiatively induced CS coefficient of the model (3) and chiral anomaly coefficent27,

$$
k_{\mu}=e^{2} b_{\mu}\left(\frac{1}{4 \pi^{2}}-\frac{1}{2} \mathcal{A}\right) .
$$

The origin of the ambiguity on radiatively induced CS coefficient is thus revealed since the chiral anomaly coefficient is ambiguous and regularization dependent. This fact was explicitly and quantitatively shown in differential regualrization 1428.

\section{Summary and Discussion}

The issue on radiatively induced Lorentz and CPT violation in quantum electrodynamics is reviewed and it has not been completely settled down yet. In this talk we emphasize how the CS term is induced from quantum correction. The physical effects it causes in classical electrodynamics were described by Jackiw 29 . Since the present astrophysical observation data has excluded the physical consequence of the CS term, thus no matter how this $k_{\mu}$ arises, either as a radiative correction induced from the fermionic sector or as free parameter set up by hand, it must vanish. Here let us discuss how to "input" this ambiguous quantum correction to compare with astrophysical observation. There are two approaches in the literature to include this CS term. One is starting from the conventional QED plus an explicit Lorentz and CPT violating in the fermionic sector, i.e.

$$
\mathcal{L}=-\frac{1}{4} F_{\mu \nu} F^{\mu \nu}+\bar{\psi}(i \not \partial-e A-m) \psi-\bar{\psi} \not b \gamma_{5} \psi=\mathcal{L}_{\mathrm{QED}}-\mathcal{L}_{b},
$$

and the CS term (2) will be induced from quantum correction with the coefficient $k_{\mu} \propto e^{2} b_{\mu}$. The other one is introducing the CS term (2) at classical level,

$$
\widetilde{\mathcal{L}}=\mathcal{L}_{\mathrm{QED}}-\mathcal{L}_{b}+\mathcal{L}_{\mathrm{CS}},
$$

pascos01: submitted to Rinton on October 30, 2018 
and the coefficient of CS term is a free parameter. The radiatively induced CS terms calculated in these two models have different meanings from the viewpoint of renormalization theory, despite that the processes of calculating CS term and the results are identical. In the framework described by $\mathcal{L}$, the induced CS term can only be considered as a radiative correction, while in the later model $\widetilde{\mathcal{L}}$, depending on the renormalization condition, the induced CS term can be cancelled by a finite counterterm and keep the classical parameter $k_{\mu}$ as the renormalized one. According to the perturbation theory of a renormalizable quantum field theory, a quantum correction calculated in certain regularization scheme, now matter how it is, finite or infinite, has no physical meaning before a renormalization procedure is implemented. Only when a renormalization condition is assigned, the quantum correction is decomposed into two parts, one part will be cancelled by certain counterterm and absorbed into the classical Lagrangian to redefine the various parameters such as mass or coupling constant, the other part is the radiative correction and reflects the observable quantum effects. Based on this fact, it can be easily seen that in the first model the induced CS term cannot be cancelled by introducing a counterterm since its has no counterpart in the classical Lagrangian, hence it can only belong to the radiative correction like chiral anomaly and anomalous magnetic moment etc. However, the induced CS term, despite of being a radiative correction, has an essential difference with anomalous magnetic moment and chiral anomaly: it can not be ambiguously fixed by the principle of a quantum field theory itself. Anomalous magnetic moment can be uniquely evaluated by a gauge invariant regularization scheme. Chiral anomaly, despite it is ambiguous, can be determined if vector gauge symmetry is required. Whereas for the induced CS term, it seems that gauge symmetry cannot dominate it 22. One may say that the CS term can be determined by the naturalness of Lorentz and CPT symmetry, but the naturalness of this space-time symmetry can only take $k_{\mu}$ to zero. The ambiguity of the induced CS term make the theory (35) awkward, since this means that the theory cannot make a definite prediction on the quantum phenomena. If we recall the explanation by Sitenko 25 on the origin of this ambiguity, this specific example seems to imply an inkling that a quantum field theory, as the most successful framework of describing subatomic physics up to now has an intrinsic deficiency in certain specific situation such as Lorentz symmetry breaking 30 . This speculation might be comprehensible, since a relativistic quantum field theory was born out of a combination between quantum theory and the theory of special relativity. If Lorentz symmetry, the algebraic soul of special relativity, collapsed, what could one expect from a relativistic quantum field model! Of course, it may not be so serious, since there exists a possible way out for the model (35), namely, considering the contribution to the CS coefficient from all the fermion species in the Standard Model 11 ,

$$
k_{\mu} \propto \sum_{i} e_{i}^{2} b_{\mu}^{i},
$$

where the sum index $i$ runs over all the leptons and quarks of the Standard Model. If $b_{\mu}^{i}$ come from the vacuum expectation value $\left\langle A_{\mu}^{5}\right\rangle$ of an axial vector gauge field, then the induced CS coefficient may vanish according to the anomaly cancellation in the Standard Model. However, as indicated by Chung et al11, it is also possible that $b_{\mu}$ may not be related to the vacuum expectation value of an axial vector field.

In contrast, the second setting (36) is more appealing in discussing the physical effects of CS term. Since CS term is put by hand at the classical level, one can introduce a finite 
counterterm to define renormalized CS coefficient 127

$$
k_{\text {ren }}^{\mu}=k_{\text {bare }}^{\mu}+k_{\text {quant }}^{\mu}+k_{\text {counter }}^{\mu},
$$

and then input $k_{\text {ren }}^{\mu}=0$ to yield to the astrophysical observation data. However, in this case, both $k_{\text {ren }}^{\mu}$ and $b_{\mu}$ are regarded as independent measurable parameters, $k_{\text {ren }}^{\mu}$ has nothing with $b_{\mu}$ and the astrophysical observation data about the vanishing of $k_{\mu}$ does not put any constraint on $b_{\mu}$ 8.27. A fine-tuning is required to get a vanishing $k_{\mu}$ and non-vanishing $b_{\mu}$ at the same time since the $\mathrm{CS}$ term can be generated from quantum correction with the coefficient proportional to $b_{\mu} 27$.

Finally, a conclusion from an investigation on the anomalous magnetic moment and Lambshift in QED with the extended fermionic sector (3) should be emphasized. It was found 31 that both the anomalous magnetic moment and Lamb shift received an additional IR divergent radiative correction proportional $b^{2}$. Furthermore, it was explicitly shown that even the IR divergence in the Lamb shift cannot be cancelled by the bremsstrahlung process as in the conventional QED31, let alone eliminating the IR divergence in the anomalous magnetic moment. Since the anomalous magnetic moment and Lamb shift are two successful symbols of QED in describing electromagnetic interaction, the IR divergence embracing them seems thus to reflect the physical inconsistency of QED with above extended fermionic sector, and therefore ruins the mechanism of generating CS term from radiative correction by introducing an explicit Lorentz and CPT violating term in the fermionic sector. Of course, there is a possibility that the calculation on the vertex correction 31 has some drawbacks, since we only expanded the $b$-exact propagators to the second order, perhaps a summation to any order of $b_{\mu}$ might erase such an IR divergence.

\section{Acknowledgment}

I would like to thank the organizers of PASCOS 2001, Professors P. Frampton and Y.J. Ng and the organizers of MRST 2001, Professors V. Elias, D.G.C. McKeon and V.A. Miransky for hospitality. I am grateful to Professor R. Jackiw for suggesting me this project and various useful discussions. I am indebted to Professors M. Chaichian, G. Kunstatter and Dr. R. González Felipe for discussion and collaboration. I would like to thank Professors G. Bonneau, L.H. Chan, V.A. Kostelecký, G. Leibbrandt and R.B. Mann for communications and discussions. I am especially obliged to Dr. M. Pérez-Victoria for his continuous discussions and comments. This work was supported in part by the Natural Sciences and Engineering Research Council of Canada.

\section{References}

1. V.A. Kostelecký and S. Samuel, Phys. Rev. Lett. 63, 224 (1989); Phys. Rev. D 39, 683 (1989); Phys. Rev. D 40, 1886 (1989).

2. B. Nodland and J. P. Ralston, Phys. Rev. Lett. 78, 3043 (1997).

3. S.M. Carroll, G.B. Field and R. Jackiw, Phys. Rev. D 41, 1231 (1990).

4. S.M. Carroll and G.B. Field, Phys. Rev. Lett. 79, 234 (1997).

5. D. Colladay and V.A. Kostelecký, Phys. Rev. D 58, 116002 (1998).

6. For a review, see talks in CPT ad Lorentz Symmetry, ed. V.A. Kostelecký (World Scientific, Singapore, 1999). 
7. S. Coleman and S.L. Glashow, Phys. Rev. D 59, 116008 (1999).

8. G. Bonneau, Nucl. Phys. B 593, 398 (2001).

9. C. Adam and F.R. Klinkhamer, Causality and Radiatively Induced CPT Violation, hep-th/0105037.

10. R. Jackiw and V.A. Kostelecký, Phys. Rev. Lett. 82, 3572 (1999).

11. J.M. Chung and P. Oh, Phys. Rev. D 60, 067702 (1999).

12. M. Pérez-Victoria, Phys. Rev. Lett. 83, 2518 (1999).

13. J.M. Chung, Phys. Lett. B 461, 318 (1999).

14. D.Z. Freedman, K. Johnson and J.I. Latorre, Nucl. Phys. B 371, 353 (1992).

15. W.F. Chen, Phys. Rev. D 60, 085007 (1999).

16. J.M. Chung, Phys. Rev. D 60, 127901 (1999).

17. L.H. Chan, Induced Lorentz-violating Chern-Simons Term in QED and Anomalous Contributions to Effective Action Expansions, hep-ph/9907349.

18. L.H. Chan, Phys. Rev. Lett. 57, 1199 (1986); M.K. Gaillard, Nucl. Phys. B 268, 669 (1986).

19. J. Schwinger, Phys. Rev. 82, 664 (1951).

20. L. Alvarez-Gaumé and E. Witten, Nucl. Phys. B 234, 269 (1983).

21. A.N. Redlich, Phys. Rev. D 29, 2366 (1984).

22. M. Chaichian, W.F. Chen and C. Montonen, Nucl. Phys. B 537, 161 (1999).

23. M. Chaichian, W.F. Chen and R. González Felipe, Phys. Lett. B 503, 215 (2001).

24. J.M. Chung and B.K. Chung, Phys. Rev. D 63, 105015 (2001).

25. Yu.A. Sitenko, One-loop Effective Action for the Extended Spinor Electrodynamics with Violation of Lorentz and CPT Symmetry, hep-th/0103215, v1.

26. C. Itzykson and J.B. Zuber, Quantum Field Theory (McGraw-Hill, New York, 1980).

27. M. Pérez-Victoria, JHEP 0104: 032 (2001).

28. P.E. Haagensen and J.I. Latorre, Ann. Phys. 221, 77 (1993); W.F. Chen, Phys. Lett. B 459, 242 (1999).

29. R. Jackiw, Comments Mod. Phys. A1 1 (1999).

30. There is a comment from Dr. M. Pérez-Victoria on this argument: According to the prescription of renormalization theory, the bare Lagrangian should consist of all the possible renormalizable terms allowed by gauge symmetry, while $\mathcal{L}_{\mathrm{CS}}$ is just such a term. Thus the Lagrangian (35) is actually incomplete and one must start from the classical Lagragian (36) to discuss the quantum correction. This is the reason why the quantum theory based on (35) is not well defined. Therefore, there should exist no problem for a quantum field theory with the breaking of Lorenz symmetry if one start from the complete Lagrangian (36). The model (35) can be understood as a special case of (36) with the renormalized $k_{\mu}$ happenning to to zero. My comprehension is that (35) and (36) are just two different model construction and they describe distinct classical electrodynamics. The quantum phenomena of these two models thus have different intepretation, for example, the radatively induced CS term in the model (35) can only be explained as radiative correction, while in (36) it can be erased by redefining the CS parameter according to the finite renormalization procedure. Of course, classical dynamics is an approximation of the corresponding quantum counterpart. In this sense, the model (36) is more appealing since it has a well defined qunatum theory. However, in some case, the model (35) rather than (36) can come from a the low-energy limit of a more fundamental theory. 
31. W.F. Chen and G. Kunstatter, Phys. Rev. D 62, 105029 (2000). 